\title{
Comunicación

\section{La formación de los ciudadanos: el papel de la televisión y la comunicación humana en la socialización política}

\author{
JUAN ENRIQUE HUERTA WONG y EDUARDO GARCÍA ${ }^{1}$
}

¿Cómo se están formando los futuros ciudadanos? ¿Cuáles son sus actitudes respecto a la participación ciudadana, el interés por los asuntos públicos? ¿Qué papel juegan agentes mediadores tales como los medios de comunicación y la familia? Este trabajo reporta los hallazgos de 1382 entrevistas por encuesta, realizadas entre marzo y mayo de 2006, en niños de sexto año de primaria de Monterrey, Saltillo y Cd. Victoria, México. Los datos se analizaron mediante modelos de análisis de trayectorias y de análisis múltiple de varianza. La evidencia apunta a la centralidad de la comunicación interpersonal para que los niños construyan su interés por los asuntos públicos y su disposición a la participación.

PALABRAS CLAVE: socialización política, análisis de trayectorias, comunicación interpersonal, televisión y educación, modelos multivariados en comunicación.
This paper addresses questions regarding how future citizenship has been built. In order to do that, this paper analyzes the role of television and interpersonal communication networks to construct political sophistication, democracy values and disposition to participation. A path analysis and a multiple analysis of variance were performed to address those questions. Results point out that the centrality of interpersonal communication networks become important to understand how citizenship has been built. Evidence permits to be optimist regarding the future of Mexican citizenship, but a final comment points out that those mexicans face sociopolitical constraints limiting that optimism.

KEY WORDS: political socialization, path analysis, interpersonal communication, television and education, multivariate analysis and communication studies.

1 Instituto Tecnológico y de Estudios Superiores de Monterrey.

Correos electrónicos: juan.huerta@mail.mcgill.ca/elmo_so79@hotmail.com 
La joven democracia mexicana se ha caracterizado por la falta de consensos. Aunque muchas voces coinciden en que, para la madurez de la democracia es necesaria la participación de los jóvenes, la evidencia disponible no permite conclusiones respecto a qué elementos inciden en esa participación. De los datos conocidos, no es fácil identificar el rol que juegan en los nuevos ciudadanos, por ejemplo, los medios de comunicación, los agentes personales de socialización, la educación, el nivel socioeconómico... en el interés por asuntos públicos, el conocimiento de los derechos, la identificación de actores y la disposición a participar en la vida pública. Tampoco se sabe mucho de la formación de esos ciudadanos en etapas previas a la mayoría de edad.

¿Cómo se están formando los futuros ciudadanos? ¿Cuáles son sus actitudes respecto a elementos clave en la democracia, tales como la participación ciudadana, la valoración de la democracia, el interés por los asuntos públicos, el conocimiento de la esfera pública? ¿Qué papel juegan agentes mediadores tales como los medios de comunicación y la familia? ¿Qué diferencias se pueden observar entre niños de distintas ciudades, sexos, niveles socioeconómicos?

La atención de los investigadores mexicanos a los procesos de socialización política ha sido aislado, pero el interés ha cobrado alguna relevancia de manera reciente. Autores como Fernández (2005), Ibarra (2003), López (2003) y Tapia (2003), han ido consolidando una línea de investigación que en México se remonta al clásico estudio de Segovia (1973). Los estudios coinciden en identificar a los niños como sujetos críticos con conocimiento más o menos sofisticado de los actores y procesos que conforman la esfera pública. Los intereses también han sido diversos. Ibarra (2003) y López (2003) han puesto el énfasis en la relación televisión-niños, mientras que Fernández (2005) y Tapia (2003), en el conocimiento de lo político y el espíritu crítico de los niños, en una línea más cercana a Segovia.

Un área negada en los estudios de comunicación y política, en México, ha sido la comunicación humana. De hecho, cuando en 2006 menudearon las críticas hacia los medios de comunicación, la tendencia fue considerar a la televisión como central en la formación de actitudes y conocimiento políticos. Esta ausencia resulta interesante porque en la literatura general sobre los procesos de socialización 
humana, se sabe que el papel de los medios de comunicación es menor ante los agentes de la comunicación interpersonal: en el niño, la familia y los maestros.

Este texto parte de las preguntas arriba planteadas, con base en una investigación empírica por encuestas realizada en tres ciudades del noreste de México, más específicamente, Monterrey, Saltillo y Ciudad Victoria.

\section{ALGUNOS CONCEPTOS DE PARTIDA}

\section{La participación política}

La discusión en torno al concepto de participación política parece inagotable. Para no abundar en la confusión, este trabajo parte del concepto de participación política en Giddens (1999) y Habermas (2006), para quienes la participación política es la constante en una sociedad democrática. Para el primero, las sociedades democráticamente avanzadas se caracterizan porque sus ciudadanos cuentan con múltiples identidades colectivas organizadas. Así pues, la participación es una consecuencia natural en tanto los ciudadanos se asumen como actores colectivos (idem, idem).

McDevitt y Kiousis (2007:1214-1230) propusieron varios modelos estructurales de covarianza en estudiantes de preparatoria de Estados Unidos, definiendo apoyo por política convencional, apoyo por activismo y activismo como las variables observadas. Sus hallazgos apoyaron la noción de que la discusión entre padres y estudiantes fue el principal predictor de participación política medida, como votar en la elección de 2004. Esta variable latente fue también observada como la principal influencia de los componentes cognitivos de involucrarse en política, e incluso de ideología política, en un modelo estructural relacionado (Kiousis, McDevitt \& Wu, 2005:756-774).

En un estudio en Nepal, Wagle (2006) encontró que el estatus socioeconómico moderó de manera importante la frecuencia del voto, implicando que el ejercicio de los derechos políticos y sociales puede estar limitado a ciertos grupos socioeconómicos en países en desarro1lo. En México, un estudio reciente encontró evidencia de que el nivel socioeconómico puede estar influyendo en la construcción de los imaginarios sociales (Buendía y Somuano, 2003:289-334). 
Desde la perspectiva de este artículo, no todas las formas de participación política tenderán a la mejora social, sino sólo aquellas que pasen por el tamiz del interés en la convivencia con esos otros, o más específicamente, por el de los valores de la democracia. Es decir, factores como el conocimiento de lo político y el interés por lo político tenderán a la mejora social en tanto pasen por el tamiz del interés de la convivencia con los otros, del entendimiento de que esos otros son nosotros. Sin el tamiz democrático, la sofisticación política, definida como la adición del conocimiento político y el interés por lo político, puede contribuir a la participación política, pero sólo por el ánimo de privilegiar a unos sobre otros.

\section{La valoración de la democracia}

El tamiz democrático resulta relevante porque es la democracia la evolución histórica que ha llevado al pueblo a gobernar colectiva y organizadamente a través del ejercicio de designar a representantes (Woldenberg y Salazar, 2001). Ese método exige la observación de ciertos valores básicos, a saber, la libertad, la justicia y la fraternidad.

En una sociedad democrática, la libertad se aplica sólo en el contexto de los límites de la misma ante los otros, es decir, en la medida en que el ejercicio de las propias libertades no afecta el de otros, de ahí que sean condicionantes de la libertad, la legalidad y la responsabilidad (idem).

La igualdad jurídica y política supone que los ciudadanos son iguales ante la ley pero no uniformes. Supone que todos los ciudadanos cuentan con todos los derechos, incluso el derecho a no ejercerlos, por ejemplo. De ahí que no sea, en términos de Salazar y Woldenberg (idem), una igualdad "igualitaria", sino respetuosa de la diversidad que guarda un conjunto complejo de seres humanos.

En cuanto a la fraternidad, significa que una sociedad puede verse como diversa pero ha de distinguirse por no verse como enemiga de sí misma, es decir, enemiga de los otros que son diferentes y que piensan diferente. En la fraternidad, por ejemplo, se sabe que la exclusión, negación o violencia hacia los diferentes no es aceptada, lo que se conoce como tolerancia.

Organizaciones civiles trabajan actualmente con programas cívicos en países como Gran Bretaña y Estados Unidos, para reforzar los 
valores de la democracia en niños de $6^{\circ}$ de primaria y de secundaria. Análisis multivariados apuntan a que estos programas han sido exitosos en utilizar medios como videos y periódicos en el reforzamiento de los valores democráticos (Kiousis, 2005). Tales análisis muestran que la exposición a estos programas cívicos han resultado poderosos predictores de disposición a la participación política a través del incremento en los valores democráticos (idem y Meirick y Wackman, 2004:1161-1214). En México, hay cierto avance en la promoción de esos valores, pero no existen análisis conocidos de su estado anterior o de su efectividad.

\section{La sofisticación política}

De acuerdo con Buendía y Somuano (2003), la sofisticación política se encuentra clásicamente asociada con la escolaridad y también con la participación política. El supuesto de partida es que la relación entre sofisticación y participación política se funda en la existencia de recursos que posibilitan la búsqueda de satisfactores no inmediatos, tales como la escolaridad y el ingreso.

Siguiendo a Buendía y Somuano (2003:289-334):

la sofisticación política o los niveles de información política son cruciales para comprender la opinión pública y el comportamiento político. Estas variables afectan la participación de manera más o menos directa, al difundir información sobre el gobierno y la política, y alientan actitudes como un sentido de responsabilidad cívica o eficacia política que predisponen al individuo a la participación política (Verba et al., 1995). [....] la sofisticación política y la edad son los dos recursos individuales más importantes que tienen los ciudadanos en términos de participación electoral. [.... Este concepto] mide el conocimiento real de los asuntos políticos y el interés que los mismos encuestados dicen tener por la política.

Estos supuestos se basan en hallazgos como los de Chaffe (1997), quien encontró que el nivel socioeconómico bajo en Estados Unidos se relacionaba con bajos niveles de escolaridad, variables que se asociaban negativamente con las cogniciones políticas, las actitudes y los comportamientos. 
Buendía y Somuano (2003) realizaron un análisis de la situación mexicana, de acuerdo con Rose y Shin (2001:331-354), para quienes una democracia de tercera ola, como la de México, corre el riesgo de haber construido una democracia electoral sin sus prerrequisitos, tales como la valoración de la misma y la presencia de una sociedad participativa. Así pues, la sofisticación política, entendida como la suma de conocimiento político, más interés por la política y/o asuntos públicos (Flores y Meyenberg, 2002), tendrá que ser función de la valoración de la democracia para contribuir en un sentido positivo con la disposición a la participación. De otro modo, sugieren Buendía y Somuano, la sofisticación política llevará a una disposición a la participación que sólo contribuirá a una democracia -o autoritarismo- incompleta. De hecho, sus conclusiones acerca de sus datos de la elección de 2000 apuntaron a que en México no existe asociación entre participación política y participación electoral, mostrando que el camino por la participación política sugería un desencanto por la participación electoral (Buendía y Somuano, 2003).

El aspecto cognitivo en la valoración de la democracia ha sido también una preocupación de los modelos de intervención en países como Gran Bretaña y Estados Unidos. De hecho, una de las razones por las que tales programas cívicos intervienen a partir del $6^{\circ}$ año de primaria, es porque se conoce que a esa edad los niños han concretado su proceso de maduración intelectual, que les llevará a "cristalizar" la disposición cívica que muestren para entonces (Sears y Valentino, 1997).

Televisión y redes de comunicación: agentes de la socialización política Para Habermas (2006), la tradición deliberativa de la democracia acentúa tanto la participación política de ciudadanos activos como las formas en que la opinión pública se forma, es decir, la socialización política. La socialización política se puede definir como el proceso por el que los individuos desarrollan concepciones de sí mismos y su mundo, así como del mundo político, incluyendo sus experiencias directas, juicios e inferencias sobre el conocimiento que en ese momento poseen. En los procesos de socialización existen agentes como la familia, los amigos, la escuela y los medios de comunicación que contribuyen al proceso de internalización de los conceptos políticos. 
De acuerdo con Gunter y McAleer (1997), el conocimiento político se relaciona con el uso de los medios masivos y, especialmente, con el consumo de noticias y de temas actuales. Esta influencia rebasa el plano de los medios tradicionales. Por ejemplo, se analizó la influencia de la información por internet en campañas políticas realizadas en 2000 y 1996, encontrando que esta fuente de información mejoraba las actitudes políticas hacia la democracia y la participación.

La televisión ha sido recurrente en las preocupaciones públicas mexicanas. Omnipresentes en las campañas políticas de este joven siglo, los anuncios políticos, la cobertura informativa y los presupuestos asignados a las televisoras han puesto sobre la mesa el tema de los efectos. No existen datos conclusivos del tema de los efectos en México. Donde hay estudios, se reducen a una ciudad o región, sin posibilidad de hablar de los mexicanos, o concretamente de los efectos de los anuncios o campañas políticas por televisión (Huerta, Garagarza y Villegas, 1999:199-226).

Lo que sí se sabe es que la televisión es el medio que más utilizan los ciudadanos para informarse de lo que pasa en la política. De acuerdo con los datos disponibles, la televisión ha ocupado de 62 a $74 \%$ de las preferencias reportadas acerca del medio para informarse de política, a nivel nacional, dato coincidente con otras investigaciones (IFE, 2003).

Un estudio reciente realizado en Estados Unidos (Paseck, 2006) encontró que los jóvenes que vieron más películas y televisión, durante una campaña electoral, mostraron un incremento en el conocimiento político y la participación. De acuerdo con este hallazgo, no fue la exposición global a la televisión, sino el uso de medios y contenidos específicos, como las noticias, lo que mostró tal incremento en el conocimiento y la participación. Este hallazgo también sugiere la hipótesis de que la edad, como predictora de conocimiento y participación política, puede ser una función de la exposición a noticias, es decir, que los jóvenes que las ven más podrían tener una disposición mayor a la participación que las personas de mayor edad que no se exponen a aquellas.

Por su parte, otros estudios (Chaffee et al., 1997; Kiousis, 2005; McLeod \& Chaffee, 2002; Sears y Valentino, 1998) han encontrado que mirar televisión interactúa con el tamaño de las redes de comunicación interpersonal para incrementar el nivel de conocimiento político 
y la disposición a la participación política. Kiousis (2005) definió el tamaño de las redes de comunicación interpersonal como la cantidad de personas y/o lugares donde una persona interactúa con otros acerca de temas políticos.

Mientras que no se conoce la dirección de la causalidad entre la mayor exposición a contenidos políticos y el tamaño de las redes de comunicación, sí se sabe que, por ejemplo, los hogares donde existe una mayor exposición a temas específicos, son también los hogares donde más se discute de política, y aquellos donde los niños conocen más de temas políticos y muestran mayor disposición a la participación. De hecho, los estudios acerca de la televisión han consistentemente mostrado que la cantidad y calidad de la exposición de los niños a la televisión se asocia con la de los padres, de manera más específica, de las madres. Lo mismo ocurre con el tema político. Sears y Valentino (1998) han mostrado que el grado de conocimiento político en niños y adolescentes se encuentra íntimamente ligado al grado de conocimiento político de los padres. La familia, han señalado McDevitt y Chaffe (2002:1-37), constituye un sistema social que mantiene el balance en distintos dominios de la interacción social, incluyendo la competencia social, incrementando además la asimilación política y generando la motivación al voto y otras formas de participación fuera del hogar. En este proceso formativo en el tema político, agregan los investigadores, la adquisición de conocimiento y opiniones va acompañada por la discusión con los padres (idem).

En cuanto a las redes de comunicación, no es mucho lo que se sabe en México. Reportó que sólo 22\% de una muestra nacional respondió que sí se platicaba de política en su casa, mientras que $70 \%$ señaló que no. Pero los datos del CESOP (Flores y Meyenberg, 2002; IFE, 2003) han permanecido a nivel descriptivo y no ha resultado posible inferir si esa discusión doméstica contribuiría a internalizar de alguna manera el conocimiento, los valores o la disposición a la participación política.

\section{Método}

Se ha planteado una pregunta general y tres preguntas de investigación: ¿Cómo se están formando los futuros ciudadanos? ¿Cuáles son sus actitu- 
des respecto a elementos clave en la democracia, tales como la participación ciudadana, la valoración de la democracia, el interés por los asuntos públicos, el conocimiento de la esfera pública? ¿Qué papel juegan agentes mediadores tales como los medios de comunicación y la familia? ¿Qué diferencias se pueden observar entre niños de distintas ciudades, sexos, niveles socioeconómicos? Las preguntas relativas a las actitudes y al papel de los agentes mediadores se responderán confrontando las hipótesis siguientes, al mismo tiempo que la pregunta relativa a las diferencias entre ciudades, sexos y NSE.

\section{Hipótesis}

H1. La exposición global a la televisión no afectará el nivel de sofisticación política, pero sí la exposición a programas de noticias.

H2. El nivel educativo de los padres influirá las redes de comunicación interpersonal de los niños.

H3. La exposición a las noticias se asociará con las redes de comunicación interpersonal.

H4. El tamaño de las redes de comunicación interpersonal predecirá el nivel de sofisticación política de los niños.

H5. El tamaño de las redes de comunicación interpersonal predecirá el nivel de valoración de la democracia.

H6. La exposición global a la televisión no afectará el nivel de valoración de la democracia ni la disposición a la participación política.

H7. La exposición a programas de noticias influirá el nivel de valoración de la democracia, y también la disposición a la participación política.

H8. El nivel de sofisticación política predecirá el nivel de valoración de la democracia y la disposición a la participación política.

H9. El tamaño de las redes de comunicación interpersonal predecirá la disposición a la participación política.

H10. La valoración de la democracia predecirá la disposición a la participación política.

\section{Participantes}

La encuesta del Proyecto Socialización Política, Televisión y Niños en el Noreste de México fue diseñada por Juan Enrique Huerta Wong 
como parte de un proyecto auspiciado por el Consejo Nacional de Ciencia y Tecnología.

Se diseñó una encuesta por etapas y por conglomerados que se levantó en escuelas primarias de Saltillo, Monterrey y Ciudad Victoria. La muestra es representativa a los conglomerados del noreste de México, a $10 \%$ de margen de error.

Las etapas fueron las siguientes:

a) Del listado general de escuelas primarias públicas y privadas, proporcionado por la Secretaría de Educación de cada estado (Coahuila, Nuevo León y Tamaulipas), se calculó y se determinó un porcentaje proporcional a la representación total de la población de escuelas de cada localidad, con respecto al universo, es decir, la suma de las escuelas ubicadas en las tres ciudades de referencia. Se determinó un total de 68 escuelas del marco muestral, tal como se muestra en la tabla 1.

b) Posteriormente, se hizo un cálculo similar para calcular la representatividad de escuelas públicas y privadas por ciudad.

c) Finalmente, cuando una escuela tuvo más de un grupo de $6^{\circ}$ grado, se sortearon los grupos también de manera aleatoria.

Cabe hacer mención que de las 68 escuelas que se tenían proyectadas en la muestra, en 4 escuelas las autoridades se negaron a que fuera aplicado el cuestionario, por lo que la muestra final se redujo a 64 escuelas.

Los criterios de elegibilidad de los participantes fueron los siguientes: niñas y niños de $6^{\circ}$ de primaria, turno matutino, de las ciudades capitales de Coahuila, Nuevo León y Tamaulipas.

Para esta muestra se seleccionaron, por muestreo aleatorio simple, escuelas públicas y privadas de los municipios de Monterrey, Saltillo y Ciudad Victoria. En total, se seleccionaron 23 escuelas públicas (17) y privadas (6) de Saltillo, 32 escuelas públicas (27) y privadas (5) de Monterrey, y 9 escuelas públicas (7) y privadas (2) de Ciudad Victoria. La tabla 1 muestra los datos de la población y la muestra de las escuelas.

Para confrontar las hipótesis, la muestra final se compuso de 588 niños de Monterrey, 551 de Saltillo y 223 de Ciudad Victoria, quienes fueron entrevistados entre marzo y abril de 2006 por los asistentes de 


\section{TABLA 1}

POBLACIÓN Y MUESTRA DE ESCUELAS, POR MÉTODO DE RACIMO

\begin{tabular}{|c|c|c|c|c|c|c|}
\hline 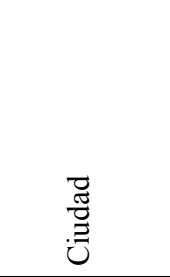 & 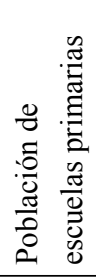 & 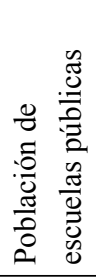 & 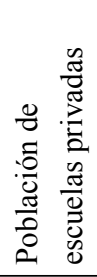 & 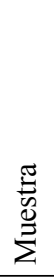 & 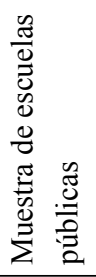 & 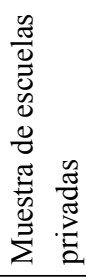 \\
\hline Cd. Victoria & 125 & $\begin{array}{c}109 \\
(87 \%)\end{array}$ & $\begin{array}{c}16 \\
(13 \%)\end{array}$ & 16 & 12 & 4 \\
\hline Saltillo & 208 & $\begin{array}{c}153 \\
(74 \%)\end{array}$ & $\begin{array}{c}55 \\
(26 \%)\end{array}$ & 23 & 17 & 6 \\
\hline Monterrey & 306 & $\begin{array}{c}207 \\
(68 \%)\end{array}$ & $\begin{array}{c}99 \\
(32 \%)\end{array}$ & 32 & 27 & 5 \\
\hline
\end{tabular}

investigación (estudiantes de maestría) Alejandra Rodríguez, Berenice Bañuelos y Eduardo García. Por el método de selección, los datos son analizados a $10 \%$ de margen de error.

En cambio, para encontrar las diferencias entre ciudades, sexos, tipo de escuela y tipo de exposición a la televisión, se construyeron variables dummy con valores tomados por percentiles. Así, los análisis para estimar estas diferencias no consideraron la muestra total de 1382 niños, sino sólo 592 ó 583, dependiendo de la variable independiente (tabla 2).

\section{Procedimiento}

La forma en como se aplicaron las encuestas tuvo toda una logística que se planeó desde las juntas celebradas por todos aquellos que operan el proyecto. Se localizaron las direcciones de las escuelas en un mapa de la ciudad, y se pusieron distintivos en cada punto del mapa para ubicar las escuelas de manera más ágil y rápida. Días antes de la aplicación en cada escuela, se llamó por teléfono a esta en horas y días hábiles, para confirmar que ahí se permitiera realizar el trabajo de campo, se habló con la directora o director, o bien con el responsable de la escuela en ese momento, para solicitarle su autorización y también para platicarle 


\section{TABLA 2}

DIFERENCIAS DE EXPOSICIÓN A LA TELEVISIÓN, VARIABLES DEMOGRÁFICAS

\begin{tabular}{|c|c|c|c|c|c|c|}
\hline & \multicolumn{2}{|c|}{$\begin{array}{c}\text { Exposición global } \\
\text { a la TV } \\
\end{array}$} & \multirow[b]{2}{*}{ Total } & \multicolumn{2}{|c|}{$\begin{array}{c}\text { Exposición } \\
\text { a las noticias }\end{array}$} & \multirow[b]{2}{*}{ Total } \\
\hline & $\begin{array}{c}\text { Mucha } \\
\text { exposición }\end{array}$ & $\begin{array}{c}\text { Poca } \\
\text { exposición }\end{array}$ & & $\begin{array}{l}\text { Mucha } \\
\text { expos. }\end{array}$ & $\begin{array}{l}\text { Poca } \\
\text { expos. }\end{array}$ & \\
\hline \multicolumn{7}{|l|}{ Ciudad } \\
\hline Monterrey & 72 & 214 & 286 & 185 & 14 & 199 \\
\hline Saltillo & 166 & 28 & 194 & 32 & 278 & 310 \\
\hline \multicolumn{7}{|l|}{$\mathrm{Cd}$. } \\
\hline Victoria & 49 & 44 & 93 & 82 & 1 & 83 \\
\hline Total & 287 & 286 & 573 & 299 & 293 & 592 \\
\hline \multicolumn{7}{|l|}{ Sexo } \\
\hline Masculino & 180 & 140 & 320 & 150 & 229 & 379 \\
\hline Femenino & 102 & 136 & 238 & 140 & 64 & 204 \\
\hline Total & 282 & 276 & 558 & 290 & 293 & 583 \\
\hline \multicolumn{7}{|c|}{ Tipo de escuela } \\
\hline Pública & 219 & 221 & 440 & 233 & 230 & 463 \\
\hline Privada & 68 & 65 & 133 & 66 & 63 & 129 \\
\hline Total & 287 & 286 & 573 & 299 & 293 & 592 \\
\hline
\end{tabular}

un poco sobre el proyecto. Se le solicitó que necesariamente tenía que ser un grupo de sexto grado.

Un estudio cualitativo realizado dos meses antes fue crucial para entender cómo se comportan los niños en el aula y cómo la comunicación con sus maestros y entre ellos mismos podía aprovecharse de mejor manera para los fines de este estudio.

Así, por ejemplo, se solicitó apoyo a los maestros para que permanecieran y colaboraran con la aplicación de la encuesta en el aula, pues los niños comprendían mejor cuando su maestra les hablaba que cuando alguno de los investigadores de campo lo hacía; este procedimiento facilitó el orden en la aplicación del instrumento y evitó demoras innecesarias y, por lo tanto, distracciones. 
Los investigadores se presentaban a los niños e indicaban: "Buenos días, el día de hoy los visitamos por que nos interesa saber sus opiniones sobre la política y queremos que por favor contesten este cuestionario, que no es un examen. No se preocupen porque no se les va a calificar; por favor saquen un lápiz y un borrador para que puedan contestar de forma adecuada"

Luego de esta explicación, se acompañó a los niños a responder las preguntas, leyendo en voz alta cada una de ellas, con el fin de que todos los niños fueran contestando al mismo tiempo. Se exhortó a estos a que leyeran a sus compañeros algunas de las instrucciones y preguntas. Al final de estas se preguntó a los niños si había dudas o si alguien no había terminado. Se resolvieron dudas personalmente para evitar que la falta de entendimiento de algunos conceptos limitara los resultados. Cuando algo en el cuestionario fue difícil de entender para algún niño, se pedían voluntarios para aclarar los conceptos. Otros niños fueron particularmente precisos al definir con claridad conceptos que al equipo de investigación costó horas de discusiones en las juntas previas, tales como injusticia, corrupción, democracia...

\section{Variables}

1. Nivel educativo de los padres: se medirá como la percepción de los niños respecto a la escolaridad del papá y de la mamá.

2. Exposición global a la televisión: índice construido de las medias de los ítem "horas al día que veo televisión de lunes a viernes", "horas al día que veo televisión los sábados y domingos" y "horas que vi televisión ayer".

3. Exposición a programas de noticias: suma de los ítem "Qué tanto ves noticias nacionales" y "Qué tanto ves noticias locales".

4. Tamaño de redes de comunicación interpersonal: suma de los ítem "Qué tanto platicas de política con tu papá", "Qué tanto platicas de política con tu mamá", "Qué tanto platicas de política con tu abuelito o abuelita", "Qué tanto platicas de política con tus compañeros de escuela", "Qué tanto platicas de política con tus primos y hermanos", "Qué tanto platicas de política con tus maestros". 
5. Nivel de conocimiento político: suma de respuestas correctas respecto a la identificación de Vicente Fox Quesada (presidente), Andrés Manuel López Obrador (candidato de PRD), Roberto Madrazo Pintado (candidato de PRI), Felipe Calderón Hinojosa (candidato de PAN), Natividad González Parás (gobernador), Roberto Campa Cifrián (candidato de Nueva Alianza) y Patricia Mercado (candidata de Alternativa).

6. Nivel de valoración de la democracia: grado de acuerdo con los valores de la democracia, expresados en los ítem a) "Siempre evito pelearme; es más fácil que yo me disculpe a que me pelee", b) "Hay otras religiones buenas además de la mía", c) "Tengo derecho a jugar si hago la tarea; si no la hago, no", d) "Frecuentemente lavo los trastes en casa", e) "Si todos elegimos a alguien para que haga los reportes, y me reporta si me porto mal, yo no me enojo", f) "Los maestros deben escuchar a sus alumnos respecto al mejoramiento de la escuela".

7. Nivel de interés por asuntos públicos: suma de interés por el voto, la pobreza, la corrupción, la democracia, la política y la seguridad.

8. Disposición a la participación política: suma de disposición propia a la participación y disposición de los padres a la participación.

9. Disposición propia a la participación: grado de acuerdo con las frases a) "Votar en esta elección será muy importante", b) "Votar es importante", c) "Me gustaría ser diputado cuando sea grande", d) "Me gustaría ser gobernador cuando sea grande", e) "Me gustaría ser jefe de grupo de mi salón”, y f) "Me gustaría unirme con otros para mejorar el país",

10. Disposición de los padres a la participación: grado de acuerdo con las frases a) "A mi papá o mamá les gustaría unirse con otros para defender el ambiente”, y b) “A mi papá o mamá les gustaría trabajar en la política".

11. Nivel de sofisticación política: suma de nivel de conocimiento político y nivel de interés por asuntos públicos.

12. Nivel socioeconómico: medido como la pertenencia a escuelas públicas o privadas.

13. Ciudad: ubicación de la escuela, ya sea Monterrey, Saltillo o Ciudad Victoria.

14. Sexo: masculino y femenino. 


\section{Validez de constructo}

Análisis factoriales exploratorios fueron realizados para probar la validez de constructo de las variables dependientes: "Nivel de interés por asuntos públicos", "Nivel de sofisticación política", "Disposición propia a la participación política", "Disposición de los padres a la participación" y "Nivel de valoración de la democracia". Todos los análisis fueron hechos de manera independiente.

La tabla 3 muestra los análisis factoriales exploratorios que fueron realizados de manera independiente para confrontar la validez de constructo de las mediciones, en la muestra total de 1382 niños y niñas de sexto año de primaria. En el caso de las variables "Nivel de sofisticación política" y "Disposición de los padres a la participación", debido a que un solo factor fue encontrado, el modelo no se rotó. Tal como lo indican las comunalidades iniciales, ambos factores son internamente consistentes y bien definidos por las variables. Respecto a las variables "Nivel de interés por asuntos públicos", "Disposición propia a la participación política" y "Nivel de valoración de la democracia", la tabla 3 muestra los datos después de la rotación varimax. En estos casos, esta tabla sólo muestra los ítem que se asumieron como parte de los constructos para análisis posteriores. Algunos de los ítems que se supusieron para formar parte del constructo no cargaron dentro del mismo factor, razón por la cual no se reportan en este trabajo. Un análisis completo de los análisis de la extracción por componentes y de las rotaciones varimax está disponible por correspondencia con el primer autor. Con estas delimitaciones, las cargas de las variables en el factor, comunalidades y porcentajes de varianza se muestran en la tabla 3.

\section{RESULTADOS}

\section{Hipótesis}

Un análisis de trayectorias (gráfica 1), una aplicación del análisis de regresión múltiple en conjunción con teoría causal fue usado para confrontar las hipótesis de investigación. Para evaluar la significancia de las relaciones propuestas en las hipótesis fueron empleadas, ecuaciones simultáneas de regresión. Más allá de la significancia estadística, se consideró que sólo los coeficientes de regresión mayores a 0.1 son 


\section{TABLA 3}

\section{VALIDEZ DE CONSTRUCTOS}

\begin{tabular}{|c|c|c|c|}
\hline Constructo & Item & $\begin{array}{l}\text { Carga en } \\
\text { el factor }\end{array}$ & $\mathrm{H}$ \\
\hline \multirow{5}{*}{$\begin{array}{l}\text { Nivel de interés por } \\
\text { asuntos públicos }\end{array}$} & Interés por la pobreza, & .713 & .509 \\
\hline & la corrupción, & .667 & .45 \\
\hline & la democracia y & .730 & .534 \\
\hline & la política & .698 & .481 \\
\hline & Porcentaje de varianza & 100 & \\
\hline \multirow{4}{*}{$\begin{array}{l}\text { Nivel de } \\
\text { sofisticación } \\
\text { política }\end{array}$} & Nivel de conocimiento político & .918 & .843 \\
\hline & Nivel de interés por asuntos & & \\
\hline & públicos & .918 & .843 \\
\hline & Porcentaje de varianza & 100 & \\
\hline \multirow{8}{*}{$\begin{array}{l}\text { Disposición propia } \\
\text { a la participación }\end{array}$} & Grado de acuerdo con las frases: & & \\
\hline & "Votar en esta elección será muy & & \\
\hline & importante" & .647 & .418 \\
\hline & "Votar es importante" & .720 & .519 \\
\hline & "Me gustaría ser jefe de grupo de & & \\
\hline & mi salón" & .552 & .305 \\
\hline & $\begin{array}{l}\text { Me gustaría unirme con otros para } \\
\text { mejorar el país" }\end{array}$ & .760 & .578 \\
\hline & Porcentaje de varianza & 100 & \\
\hline \multirow{7}{*}{$\begin{array}{l}\text { Disposición de } \\
\text { los padres a la } \\
\text { participación }\end{array}$} & Grado de acuerdo con la frase: & & \\
\hline & “A mi papá o mamá les gustaría & & \\
\hline & unirse con otros para defender el & & \\
\hline & ambiente & .749 & .561 \\
\hline & “A mi papá o mamá les gustaría & & \\
\hline & trabajar en la política" & .749 & .561 \\
\hline & Porcentaje de varianza & 100 & \\
\hline \multirow{6}{*}{$\begin{array}{l}\text { Nivel de valoración } \\
\text { de la democracia }\end{array}$} & Grado de acuerdo con la frase: & & \\
\hline & "Tengo derecho a jugar si hago la & & \\
\hline & tarea; si no la hago no" & .608 & .324 \\
\hline & "Si todos elegimos a alguien para & & \\
\hline & que haga los reportes, y me reporta & & \\
\hline & si me porto mal, yo no me enojo" & .588 & .222 \\
\hline
\end{tabular}




\begin{tabular}{|c|c|c|c|}
\hline \multirow[t]{9}{*}{ Constructo } & Ítem & $\begin{array}{l}\text { Carga en } \\
\text { el factor }\end{array}$ & $\mathrm{H}$ \\
\hline & $\begin{array}{l}\text { "Frecuentemente lavo los trastes } \\
\text { en casa" }\end{array}$ & .569 & .294 \\
\hline & "Siempre evito pelearme; es más & & \\
\hline & fácil que yo me disculpe a que me & & \\
\hline & pelee" & .542 & .346 \\
\hline & "Los maestros deben escuchar a sus & & \\
\hline & alumnos respecto al mejoramiento & & \\
\hline & de la escuela" & .471 & .370 \\
\hline & Porcentaje de varianza & 100 & \\
\hline \multirow{5}{*}{$\begin{array}{l}\text { Disposición a } \\
\text { la participación } \\
\text { política }\end{array}$} & Disposición de los padres a la & & \\
\hline & participación & .809 & .654 \\
\hline & Disposición propia a la & & \\
\hline & participación & .809 & .654 \\
\hline & & 100 & \\
\hline
\end{tabular}

reportables en apoyo de una hipótesis de investigación u ofreciendo información relevante.

Disposición a la participación. Un análisis de regresión múltiple incluyó los efectos de la "Exposición global a la televisión", la "Exposición a programas de noticias", el "Nivel de sofisticación política", el "Tamaño de las redes de comunicación interpersonal" y la "Valoración de la democracia" en la "Disposición a la participación". Los resultados de este análisis permitieron una $R^{2}$ significativa de $.227, F(4,706)=$ $51.715, p<.001$. En concordancia con lo descrito en el párrafo anterior, sólo se muestran en la gráfica 1 las relaciones mayores a 0.1 , es decir, la "Valoración de la democracia" $(b=.402, p<.001)$ y la "Exposición a noticias" $(b=.101, p<001)$.

Estos hallazgos soportan la hipótesis 10, que estableció que la "Valoración de la democracia" predecirá a la "Disposición a la participación política". Asimismo, se soporta parcialmente la hipótesis 7, pues la "Exposición a noticias" predice, si bien débilmente, la Disposición a la participación política. La hipótesis 6 también fue parcialmente apoyada, pues la "Exposición global a la televisión" no influyó en la "Disposición a la participación". En cambio, no se apoyaron las hipótesis 8 y 9, que pre- 
dijeron que las "Redes de comunicación interpersonal" y el "Nivel de sofisticación política" predicen la "Disposición a la participación política".

Valoración de la democracia. Un análisis de regresión múltiple incluyó los efectos de "Tamaño de las redes de comunicación interpersonal", la "Exposición global a la televisión", la "Exposición a programas de noticias" y el "Nivel de sofisticación política en la Valoración de la democracia". Los resultados de este análisis permitieron una $R^{2}$ significativa de $.191, F(4,768)=7.285, p<.01$. Tal como se ha dicho antes, sólo se muestran en la Gráfica 1 las relaciones mayores a 0.1 , es decir, el "Nivel de sofisticación política" $(b=.107, p<.01)$ y las "Redes de comunicación interpersonal" ( $b=.154, p<.05)$.

Estos hallazgos permiten soportar la hipótesis 5, pues las "Redes de comunicación interpersonal" predicen la "Valoración de la democracia". La hipótesis 6 también fue apoyada; esta predijo que la Exposición global a la televisión no se relaciona con la "Valoración de la democracia" ni con la "Disposición a la participación política". También soporta la hipótesis 7, que establece la relación entre la "Exposición a noticias" con la "Valoración de la democracia" y la "Disposición a la participación". La hipótesis 8 resultó parcialmente soportada, pues el "Nivel de sofisticación política" predice la "Valoración a la democracia".

Sofisticación política. Un análisis de regresión múltiple incluyó los efectos de "Tamaño de las redes de comunicación interpersonal", la "Exposición global a la televisión", la "Exposición a programas de noticias" y el "Nivel educativo de los padres" en el "Nivel de sofisticación política". Los resultados de este análisis permitieron una $R^{2}$ significativa de $.275, F(4,772)=15.786, p<.01$. Tal como se ha dicho antes, sólo se muestran en la gráfica 1 las relaciones mayores a 0.1 , es decir, la "Exposición a noticias" $(b=.196, p<.01)$, las "Redes de comunicación interpersonal" $(b=.134, p<.05)$ y la Educación $(b=.183, p<.05)$.

Estos hallazgos soportan la hipótesis 1, la cual predijo que la "Exposición a programas de noticias", pero no la "Exposición global a la televisión", afectaría el "Nivel de sofisticación política". También se apoyó la hipótesis 4, que estipuló que el "Tamaño de las redes de comunicación interpersonal" predecirá el "Nivel de sofisticación política".

Relaciones entre las variables independientes. Se realizó un análisis de regresión para determinar el efecto del nivel educativo de los pa- 
dres en la conversación de temas políticos. La hipótesis 2, que enunció que el nivel educativo de los padres influirá las redes de comunicación interpersonal de los niños no fue apoyada. Un análisis de correlación divariada, en cambio, determinó la asociación entre la exposición a las noticias y la frecuencia con que los niños platican acerca de temas políticos. La hipótesis 3, estableciendo tal asociación, resultó apoyada $(R$ $=.204, p<.01)$.

\section{GRÁFICA 1}

ANÁLISIS DE TRAYECTORIAS

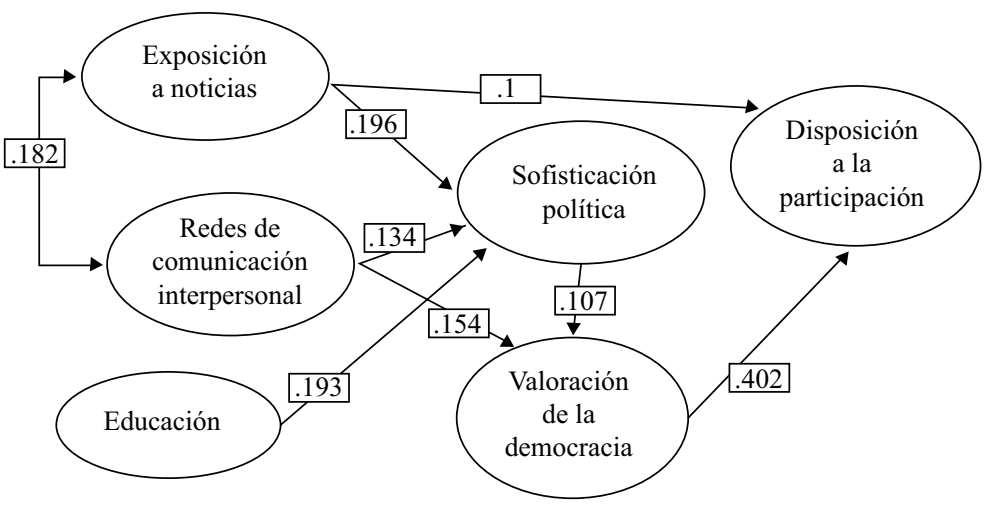

Diferencias entre ciudades, sexos y tipo de escuela

Un análisis de varianza múltiple de dos vías (MANOVA) fue conducido sobre tres variables dependientes, a saber, "Nivel de sofisticación política", "Valoración de la democracia" y "Disposición a la participación". Las variables independientes fueron ciudades (Monterrey, Saltillo, Cd. Victoria), sexos (niños, niñas), tipo de escuela (pública, privada), exposición global a la televisión $(\mathrm{H}, \mathrm{L}$,$) y exposición a las noticias (\mathrm{H}, \mathrm{L}$,$) . Para maximizar$ las diferencias en términos de la exposición a la televisión, se construyó una variable dummy con dos valores, personas que se exponen mucho y personas que se exponen poco a la televisión (heavy \& light viewers). Para hacerlo, se tomaron a las personas sobre el percentil 75 (mucho) y debajo del percentil 25 (poco) de la distribución. 
El modelo completo resultó significativo. Por motivos de claridad, la tabla 4 sólo muestra las relaciones univariadas. El análisis completo está disponible bajo correspondencia con el investigador principal.

Como parte del análisis del modelo completo, análisis de varianza de una vía fueron conducidos en cada variable dependiente para determinar el locus del efecto multivariado estadísticamente significativo de la ciudad, tipo de escuela, sexo, exposición global a la televisión y exposición a las noticias de los informantes.

En la tabla 4 se puede apreciar que la ciudad donde radican los informantes sí produjo efectos significativos en las tres variables dependientes. Una inspección de las medias de grupo sugiere que los niños y niñas de Saltillo, en realidad, tuvieron indicadores más altos de "Nivel de sofisticación política", pero más bajos en términos de "Valoración de la democracia" y "Disposición a la participación" que los niños de Monterrey y Cd. Victoria. No se reportaron diferencias entre los niños de Monterrey y $\mathrm{Cd}$. Victoria en ninguna de las variables dependientes.

En la tabla 4 se puede apreciar que el tipo de escuela donde estudian los informantes produjo efectos significativos en el "Nivel de sofisticación política" y la "disposición a la participación, pero no en la valoración de la democracia. Una inspección de las medias de grupo sugiere que los niños y niñas que estudian en escuelas privadas en realidad tuvieron indicadores más altos de "Nivel de sofisticación política" que los niños que estudian en escuelas públicas, aunque más bajos en términos de "Disposición a la participación". Hasta aquí los datos sugieren que los niños que tenían más conocimiento y se interesaban más en asuntos públicos, también mostraban cierto desencanto hacia los mismos asuntos, mostrando una menor disposición a participar que quienes saben y se interesan menos en la esfera pública.

En la tabla 4 se puede apreciar que el sexo de los informantes produjo efectos significativos en términos del "Nivel de sofisticación política" y la "Valoración de la democracia", pero no en la "Disposición a la participación". Una inspección de las medias de grupo sugiere que las niñas, en realidad, son preciudadanas más sofisticadas y con una mayor valoración de la democracia que los niños.

En la tabla 4 se puede apreciar que la "Exposición global a la televisión" sí produjo efectos significativos en términos de la "Valoración 
de la democracia", pero no en el "Nivel de participación" ni en la "Disposición a la participación". Una inspección de las medias de grupo sugiere que las personas que se exponen más a la televisión valoran menos la democracia que quienes se exponen poco.

En la tabla 4 se puede apreciar que la "Exposición a las noticias" sí produjo efectos significativos en las tres variables dependientes. Una inspección de las medias de grupo sugiere que los niños y niñas que reportan ver más noticias, en realidad tuvieron mayores puntajes en términos de "Nivel de sofisticación política", "Valoración de la democracia" y "Disposición a la participación" que quienes reportaron ver menos noticias.

\section{TABLA 4}

ANÁLISIS MÚLTIPLE DE VARIANZA, RESULTADOS UNIVARIADOS

\begin{tabular}{lccc} 
& Sofisticación & Valoración & Disposición \\
\hline Ciudad & & & \\
Monterrey & 12.11 & $10.44^{* *}$ & 17.87 \\
Saltillo & $15.29^{* *}$ & 10.01 & $15.08^{* *}$ \\
Cd. Victoria & 11.84 & 10.47 & 17.92 \\
\hline Tipo de escuela & & & \\
Pública & $12.63^{*}$ & 10.33 & $17.20^{*}$ \\
Privada & 13.75 & 10.34 & 16.93 \\
\hline Sexo & & & \\
M & $13.03^{*}$ & $10.78^{* *}$ & 17.52 \\
H & 12.83 & 9.98 & 16.81 \\
\hline Global & & & \\
H & $=$ & $10.25^{* *}$ & $=$ \\
L & & 10.50 & \\
\hline Noticias & & & $18^{* *}$ \\
H & $13.78^{* *}$ & $10.84^{*}$ & 16.45 \\
L & 12.15 & 10.21 & \\
\hline
\end{tabular}

$* \mathrm{p}<.05 \quad * * \mathrm{p}<.01$

\section{Discusión}

Para iniciar esta sección es preciso hacer algunas delimitaciones. El análisis de los datos, en este trabajo, está descrito a nivel exploratorio y 
es necesario tomar los resultados con cautela. Los coeficientes de regresión y de correlación no son grandes, en general. Esto también sugiere alguna falta de consistencia en los constructos. Análisis más sofisticados como los modelos de ecuaciones estructurales tienen que ser realizados con la finalidad de comparar modelos más parsimoniosos. Otra área de oportunidad consiste en la comparación de submuestras. Esto se debe a que los modelos han sido tomados con cierta ligereza, razón por la cual se ha tenido que conferir un margen de error de $10 \%$. Una comparación de submuestras reforzará la validez externa de los hallazgos, permitiendo conclusiones más puntuales acerca de la hipótesis que ocupa a este texto.

Con toda la cautela que merecen los datos, se puede explorar una respuesta a la pregunta: ¿cómo se están formando los futuros ciudadanos? Para abundar en esta respuesta, en principio hay que responder, con base en los datos mostrados, las preguntas de investigación.

Participación ciudadana, valoración de la democracia y sofisticación política. El análisis de trayectorias mostró que la valoración de la democracia es el principal predictor de disposición a la participación ciudadana. De hecho, es la relación más fuerte en el modelo. En contraste, el nivel de sofisticación política, entendido como la suma de cuánto se sabe y cuál es el grado de interés en asuntos públicos, no fue un predictor sustancial de la disposición a la participación ciudadana. El nivel de sofisticación política, en cambio, contribuye a explicar la valoración de la democracia. Es relevante que, al menos entre los niños de la muestra, la disposición a participar cruza por el tamiz de la valoración de la democracia. Relevante porque, desde la taxonomía clásica de cultura política (ver Almond y Verba, 1963; Ibarra, 2003), no todas las culturas políticas son democráticas. En sentido estricto, la disposición a la participación pudiera tener una génesis no democrática. Así que se puede ser optimista respecto a la cultura política de los preciudadanos (en términos de Fernández Poncela, 2005) de la muestra en cuestión, pues esta cultura política muestra rasgos interesantes de democratización.

La televisión y las redes de comunicación interpersonal. ¿Qué causa las relaciones discutidas en el párrafo anterior? Los datos del análisis de trayectorias sugieren que las redes de comunicación interpersonal, o la frecuencia con que los niños platican con familiares, maestros y com- 
pañeros de clase acerca de temas políticos, juega un rol más importante que la exposición a la televisión, e incluso la educación, en explicar el nivel de sofisticación política y la propia valoración de la democracia. Esto es relevante porque abre la puerta a la posibilidad de actuar en el plano de lo personal ante lo estructural. En el contexto de la crítica hacia los medios de comunicación durante las elecciones federales de 2006, y aun después de esas críticas, se partió del principio de la centralidad de la televisión en la vida cotidiana de México. Una serie de estudios (Huerta, Garagarza y Villegas, 1999; Huerta, 2007) se suman a la noción de que el impacto de la televisión es menor, al menos entre los habitantes del noreste de México, frente a la cantidad y calidad de la interlocución humana. En otras palabras, la exposición a la televisión aparece siempre mediada por los agentes, en este caso concreto, de socialización de los niños, ya sean familiares o maestros (los compañeros de clase no aparecieron como interlocutores en asuntos políticos). Esta mediación no pasa, tampoco, por el nivel de educación de los padres, con lo que se puede ser optimista para sugerir que tampoco es un asunto de clase o de contar con más recursos de alguna manera, que la propia atención que se confiere a los niños y a los temas públicos. Otra vez, la comunicación humana ha sido un área extrañamente negada en México, y este estudio se suma a otros (idem; idem) donde la interlocución humana aparece como una variable más importante que la propia exposición a la televisión para construir la realidad social, al menos, en el noreste de México.

La formación de los (pre)ciudadanos. La noción de preciudadanía es relevante porque en sexto año de primaria, de acuerdo con los pedagogos (Fernández, 2005; McDevitt y Kiousis, 2005), los niños han construido sus bases cognoscitivas y de valores. Aunque existe una discusión en términos de los efectos de largo plazo de tales bases (idem), hay también cierto consenso en el sentido de que la evidencia disponible no permite desconfiar de tales efectos. En otras palabras, los datos disponibles establecen que no existen argumentos fuertes en contra de la noción de que las actitudes, consensos y diferencias que los niños tienen en sexto año de primaria prevalezcan en el largo plazo.

Si es así, se puede ser optimista respecto al futuro. Los preciudadanos de la muestra estarán listos para votar en 2012 con bases sólidas en 
términos de la valoración de la democracia. La relevancia de la comunicación interpersonal en la formación de sofisticación política y valoración de la democracia sugiere que la intervención a edades tempranas es la variable clave en el reforzamiento de una cultura cívica y una mayor disposición a la participación política. Sin embargo, no resulta recomendable dejar al libre albedrío la escolaridad si lo que se pretende es que los contenidos educativos hacen una diferencia. La calidad de esa escolaridad resulta determinante para un tema crucial, como los factores que motivan la disposición a la participación política. En algunos países, como Estados Unidos y Gran Bretaña, el éxito de la intervención educativa para fomentar la interacción y así contribuir a la formación de ciudadanos ha sido probada (Buckingham, 2000; McDevitt y Kiousis, 2005). En México, si bien el IFE ha trazado tal necesidad, no existen esfuerzos consistentes en esta materia, pese a que los partidos políticos reciben fondos etiquetados con estos fines.

Un último comentario, hablando de partidos políticos, respecto a la formación de ciudadanos. Es importante que el contexto no disuada a estos respecto a la valoración de la democracia. La relación entre sofisticación política y disposición a la participación desde la comparación de las medias grupales sugiere que a medida que un preciudadano conoce más, se interesa menos en participar en la vida pública. Después de todo, el análisis de los factores del constructo "Disposición" mostró que los niños están interesados en participar en la política en un sentido amplio, pero no en la política formal. Estos hallazgos han sido consistentes, por ejemplo, con el trabajo de Fernández (2005). Más ampliamente, los datos de la Encuesta de Cultura Política han mostrado que la ciudadanía está harta del secuestro de la vida pública por parte de los partidos políticos. Más imaginación sociológica es necesaria en el diseño de investigación e intervención social para develar los mecanismos de formación de ciudadanos, pese a la realidad sociopolítica que se vive en México.

\section{Bibliografía}

ALMOND, G. A. y Verba, S. (1963) The civic culture : political attitudes and democracy in five nations, Princeton, N.J. : Princeton University Press. 
BUCKINGHAM, D. (2000) The making of citizens: young people, news and politics, Londres y Nueva York: Routledge.

BuENDÍA, J., Somuano, F. (2003) Participación electoral en nuevas democracias: le elección presidencial de 2000 en México, Política y Gobierno, 10.

Comisión Estatal Electoral (2006) Estadísticas generales, disponible, el 20 de septiembre de 2006, en www.cee_nl.org.mx.

EvelAND, W., McLeod, J., Horowitz, E. (1998) "Communication and age in childhood political socialization: an interactive model of political development", Journalism and Mass Communication Quarterly, 75 (4).

FERNÁNDEZ, Anna M. (2005) Infancia, adolescencia y politica en México, Porrúa/Instituto Electoral del Distrito Federal.

FLORES, J. y Meyenberg, Y. (2002) Ciudadanos y cultura de la democracia, consultado en septiembre, 5, 2006 en www.ife.mx

GIDDENS, A. (1999) La tercera vía, Madrid: Taurus.

GUNTER, B. y McAleer, J. (1997) Children and orkisión, Nueva York: Routledge.

Habermas, J. (2006) Political communication in media society - does democracy still enjoy an epistemic dimension? The impact of normative theory on empirical research, $56^{\text {th }}$ Annual Conference of the internacional Communication Association, disponible el 4 de septiembre de 2006, en http://www.icahdq.org/Speech_by_Habermas.pdf.

HUERTA J.,Garagarza I., Villegas L. (1999) "Percepción de la violencia en televidentes del área metropolitana de Monterrey”, Comunicación y Sociedad, 36.

HUERTA Wong, J. E. (2007) "El análisis del cultivo y las construcciones sociales del género en televidentes", en Orozco, G. (ed.), Un mundo de visiones. Interacciones de las audiencias en múltiples escenarios mediáticos y virtuales, México: Instituto Latinoamericano de Comunicación Educativa.

IBARRA, A. M. (2003) Televisión y socialización politica de escolares de la zona metropolitana de Guadalajara, tesis doctoral, Universidad de Guadalajara.

INEGI (2003) Estadísticas a propósito del día mundial de la población datos nacionales, disponible, el 15 de septiembre de 2006, 
en http://www.sep.gob.mx/work/resources/LocalContent/15105/1/ 2003\%20poblacion.pdf

IFE (2003) La naturaleza del compromiso cívico: capital social y cultura política en México, México: Instituto Federal Electoral.

JosH, P., Kenski K., Romer D. (2006) “America's youth and community engagement", Communication Research, 33 (3).

LÓPEZ, G. (2003) Televisión y socialización política de los niños en Monterrey y Juchitán, tesis de maestría: Tecnológico de Monterrey.

KIOUSIS, S. McDevitt, M., Wu, X. (2005) "The genesis of civic awareness: agenda setting in political socialization", Journal of Coтmunication, 55.

MARTínEZ, E. (2004) Seminario “Democracia, Política y Estado", Relatoría, consultado en septiembre, 17, 2006 en http://democracyreport.undp.org/Downloads/SeminarioMexicoConclusiones.pdf.

MCComBS M., Shaw D., Weaver D. (1997) Communication and democracy: exploring the intellectual frontiers in agenda-setting theory. Nueva Jersey: Lawrence Erlbaum Associates.

MCDEVITT, M; y Chaffe, S. (2002) "The family in a sequence of political activation: why civic interventions can succeed", Journalism and Communication Monographs, 4.

- y Kiousis (2007) "The red and blue of adolescence: origins of the compliant voter and defiant activist”, American Behavioral Scientist, 50.

- y Kiousis, S. (2005) Experiments in political socialization: kids voting USA as a model for civic education reform, final report to the John S. and James L. Knight Foundation, disponible el 12 de noviembre de 2007, en http://www.civicyouth.org/PopUps/WorkingPapers/WP49McDevitt.pdf

MEIRICK P., Wackman D. (2004) "Kids voting and political knowlwdge: narrowing gaps, informing votes", Social Science Quarterly, 85 (5).

MORTIMORE, R. y Tyrrell, C. (2004) “Children's acquisition of political opinions", Journal of Public Affaires, 4 (3).

PUTNAM, R. (2000) Bowling alone: the collapse and revival of American community, New York: Simon \& Shuster.

Rose, R. y Shin, D. C. (2001) "Democratization backwards: the problem of third-wave democracies", British Journal of Political Science, (31). 
Secretaría de Gobernación (2005) Encuesta Nacional de Cultura Política de 2005, consultado en septiembre, 12, 2006 en_http://www. gobernacion.gob.mx/index.php.

SEARS, D. O., \& Valentino, N. A. (1997) "Politics matters: political events as catalysts for preadult socialization", American Political Science Review, 91.

TAPIA, E. (2003) Socialización política y educación cívica en los niños, Querétaro, México: Instituto Mora.

WAGLE, U.R. (2006) "Political participation and civic engagement in Kathmandu: an empirical analysis with structural equations", International Political Science Review, 27.

Woldenberg J., Salazar L. (2001) Principios y valores de la democracia, México: IFE.

Esta investigación fue parcialmente apoyada por el Fondo de Investigación Básica SEP-CONACyT y la Cátedra de Investigación en Medios de Comunicación del Tecnológico de Monterrey. Juan Enrique Huerta quiere agradecer al Dr. José Carlos Lozano, director del Centro de Investigación en Comunicación e Información del Tecnológico de Monterrey por su apoyo económico, moral e intelectual, y también reconoce su deuda con el Dr. Philip Oxhorn, director del Centre for Developing-Area Studies de McGill University, por facilitar los recursos necesarios para terminar con este proyecto. Los autores agradecen los comentarios de dos revisores "ciegos", quienes ayudaron a mejorar el texto de manera significativa.

Fecha de recepción: 09/11/2007. Aceptación: 21/04/2008. 